\title{
Multi-Messenger observations of the $\gamma$-ray blazar 4FGL J0658.6+0636 consistent with an IceCube high-energy neutrino
}

\section{Raniere de Menezes, ${ }^{a}{ }^{*}$ Sara Buson ${ }^{b}$ and collaborators on behalf of the Fermi-LAT, H.E.S.S., MAGIC, VERITAS, ZTF, and TELAMON Collaboration}

(a complete list of authors can be found at the end of the proceedings)

\author{
${ }^{a}$ Universidade de São Paulo, Departamento de Astronomia, Rua do Matão, 1226, São Paulo, SP \\ 05508-090, Brazil \\ ${ }^{b}$ Institut für Theoretische Physik and Astrophysik, Universität Würzburg, D-97074 Würzburg, Germany \\ E-mail: raniere.m.menezes@gmail.com
}

\begin{abstract}
The detection of cosmic neutrinos has raised many new questions in astroparticle physics, among the most compelling of which is the identification of cosmic neutrino emitters. After more than a decade of IceCube operations, the most promising neutrino astrophysical association remains the very-high-energy (VHE, > $100 \mathrm{GeV}$ ) blazar TXS 0506+056. Recently, on November 14, 2020 the IceCube observatory reported the detection of a well-reconstructed high-energy neutrino event, IceCube-201114A, with a high probability of being astrophysical. Within the $90 \%$ IceCube201114A localization region only one known $\gamma$-ray ( $>100 \mathrm{MeV}$ ) source is found. This is 4FGL J0658.6+0636, associated with the blazar NVSS J065844+063711. In these proceedings we present results from the rich multi-messenger campaign triggered by the IceCube-201114A neutrino detection, which has allowed us to collect simultaneous and quasi-simultaneous data for the $\gamma$-ray source potentially associated with the neutrino. NVSS J065844+063711 is a previouslyknown blazar with broadband properties resembling a high-synchrotron-peaked object, making it a promising $\mathrm{TeV}$ emitter. Indeed, the detection of very high-energy (VHE) photons (i.e., > 100 $\mathrm{GeV}$ ) by the Fermi Large Area Telescope provides the first evidence of such emission from this object. It makes NVSS J065844+063711 the second VHE object found within the $90 \%$ confidence region of a well-reconstructed, high-energy IceCube event.
\end{abstract}

$37^{\text {th }}$ International Cosmic Ray Conference (ICRC 2021)

July 12th-23rd, 2021

Online - Berlin, Germany

\footnotetext{
${ }^{*}$ Presenter
} 


\section{Introduction}

The detection of a diffuse background of astrophysical neutrinos with the IceCube Neutrino Observatory in 2013 opened a new window to the sky [1,2]. Neutrinos are unambiguous tracers of cosmic-ray acceleration sites, as they are able to escape unimpeded from dense astrophysical environments generally opaque to other particles [3]. The interactions of relativistic protons and heavier nuclei with the gas and background light near these sites can produce unstable particles, like pions and kaons, which quickly decay into secondary particles, including neutrinos [2].

In 2017, an IceCube neutrino (IceCube-170922A) of high probability of being astrophysical was detected in both spatial and temporal coincidence with the $\gamma$-ray flaring blazar TXS 0506+056 [4], providing the first direct evidence of cosmic-ray acceleration in blazars' jets. This discovery prompted a search in the archival IceCube data revealing an excess of low-energy neutrinos with respect to the atmospheric background positionally consistent with IceCube-170922A. The lowenergy neutrino excess was observed $2 \sim 3$ years before the detection of IceCube-170922A and is likely associated with the $\gamma$-ray blazar, i.e., with a significance of $\sim 3.5 \sigma$ [5]. However, during the epoch of the neutrino excess, the blazar displayed an electromagnetic emission compatible with a low activity state in radio, optical and even the GeV energy band [6, 7]. The putative detection of neutrino emission suggests the presence of hadronic $\gamma$-ray emission from blazars, which can be generated by synchrotron radiation of relativistic protons [8] or photo-hadronic interactions with the ambient photon field [9]. The spectral energy distribution (SED) of TXS 0506+056is fairly well described by leptonic models, suggesting that if hadronic processes are at work, their contribution to the overall observed emission is not dominant $[10,11]$.

On November 14, 2020, the IceCube observatory reported the detection of another wellreconstructed high-energy neutrino, IceCube-201114A, which was found to be spatially consistent with the $\gamma$-ray blazar 4FGL J0658.6+0636, associated with the high-synchrotron-peaked object NVSS J065844+063711 [12]. The $\gamma$-ray observations performed with the Fermi Large Area Telescope (LAT) show no enhanced activity of this blazar during the neutrino arrival time. In this contribution, we present the ensemble of the multimessenger observations of NVSS J065844+063711. We include archival and new observations quasi-simultaneous to the neutrino arrival, spanning several energy bands from radio to VHE $\gamma$-rays.

\section{IceCube-201114A}

On November 11, 2020, at 15:05:31.96 UTC, the IceCube observatory reported the detection of a "gold" neutrino ${ }^{1}$, i.e. a high-energy neutrino track event (IceCube-201114A) with a $>50 \%$ likelihood to be of astrophysical origin. It had a reconstructed direction of right ascension (RA) $=105.25_{-1.12^{\circ}}^{\circ+1.28^{\circ}}$ and declination $(\mathrm{Dec})=6.05_{-0.95^{\circ}}^{\circ+0.95^{\circ}}$ (90\% containment, statistical errors only). Its estimated energy is $\sim 214 \mathrm{TeV}^{2}$. No neutrino candidate events were recorded by the ANTARES observatory within the $90 \%$ error box of the IceCube event during a $+/$ - one day time-window centered on the IceCube event time [30].

\footnotetext{
${ }^{1}$ https://gcn.gsfc.nasa.gov/doc/IceCube_High_Energy_Neutrino_Track_Alerts.pdf

${ }^{2}$ Astrophysical signalness $=0.56$. More details about this event can be found here: https: //gcn.gsfc . nasa .gov/ notices_amon_g_b/134698_40735501.amon
} 


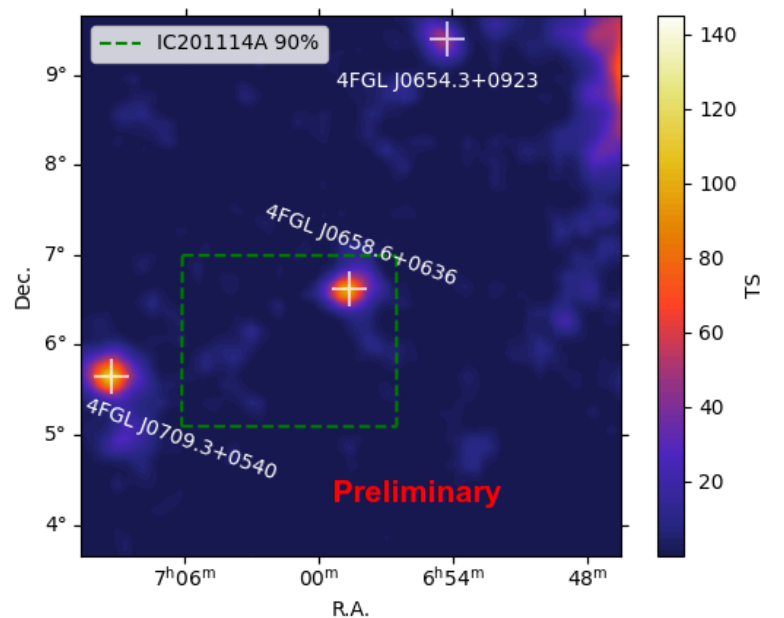

Figure 1: Fermi-LAT TS map centered on 4FGL J0658.6+0636 (a.k.a. NVSS J065844+063711), which is the only $\gamma$-ray source within the $\sim 90 \%$ containment region of IceCube-201114A, given by the dashed contour. This map is based on $\gamma$-ray observations in the energy range $0.1-500 \mathrm{GeV}$, integrating LAT data from August 2008 to February 2021. Only the extended sources and diffuse background are modelled, hence the point-sources in the region are highlighted.

At $\gamma$-ray energies, the continuous all-sky monitoring by Fermi-LAT revealed only one $\gamma$-ray source significantly $(>5 \sigma)$ detected within the IceCube-201114A error region [12], as shown in Figure 1. According to the Fermi-LAT Fourth Source Catalog [4FGL; 14] this is the $\gamma$-ray object 4FGL J0658.6+0636, associated with the blazar NVSS J065844+063711. The well-reconstructed neutrino event prompted several follow-up observations in the very-high-energy (VHE) $\gamma$-ray [29], $\mathrm{X}$-ray, optical, and radio bands focused on the candidate neutrino $\gamma$-ray counterpart. In $\S 3$ we summarize initial results from this multi-messenger campaign, while the full information will be reported in [22].

\section{Observations}

We collected archival data from the ASI Space Science Data Center (SSDC) ${ }^{3}$ and performed new observations of NVSS J065844+063711 at $\gamma$-ray, X-ray, ultraviolet, optical, and radio wavelengths, quasi-simultaneous with the neutrino arrival time. In the following section we describe the observations at VHE by H.E.S.S., MAGIC and VERITAS and at MeV-GeV energies by Fermi-LAT. For a description of the full acquired dataset we refer the reader to [22].

\subsection{Very high-energy $\gamma$ rays}

The H.E.S.S. observations presented here were obtained in two epochs to increase the overlap with the MWL campaign. The first observation period took place between the 19th and 26th November 2020, while the second period covered the 10th to 12th December 2020. After data and calibration quality selection a total of 14.5 hours of data are available for analysis. Here we combined the full dataset and reconstruct the arrival direction and energies of the $\gamma$-ray events using a log-likelihood optimization comparing the shower images recorded by the four 12-meter telescopes to a semi-analytical model of air showers [23]. A cross-check with an independent calibration and analysis chain, using the Image Pixel-wise fit for the Atmospheric Cherenkov Telescopes ImPACT reconstruction method [24], shows consistent results. We define a circular region-of-interest with a radius of $0.12^{\circ}$ centered on the optical position of NVSS J065844+063711. The background level in this region was determined using the standard "ring background" technique [25] allowing the background level to be derived from the data itself.

\footnotetext{
3https://tools.ssdc.asi.it/
} 
MAGIC observed NVSS J065844+063711 from the 16th to the 25th November 2020, for a total exposure of 7.5 hours. After quality cuts, two nights (16th and 20th) were excluded from the data set, resulting in 6.3 hours of available data. The analysis was performed using MARS, the MAGIC Analysis and Reconstruction Software [26]. No signal was detected either for any single night of observation or for the whole stacked data sample. During the computation of upper limits no negative fluctuations were allowed [4].

VERITAS observed NVSS J065844+063711 from the 15th to the 19th November 2020 for a total exposure of approximately 8 hours. After quality cuts, 7 hours of data were available. The analysis was performed using Eventdisplay [27] with the background estimated through the standard "ring background" technique. Cross-check analysis was performed with an independent software package (VEGAS) [28].

Given the lack of detection in any of the observations obtained by the VHE instruments, differential upper limits were computed according to a common configuration using the Rolke method [31], considering a confidence level of $95 \%$ and a global $30 \%$ systematic uncertainty and assuming a power law spectrum with photon index $\alpha=-2.5$.

\subsection{Fermi-LAT}

We select Fermi-LAT data within a $15^{\circ} \times 15^{\circ}$ region-of-interest (ROI) centered on the optical position of NVSS J065844+063711. The period of observations spans 12.5 years, ranging from 4th August 2008 to 10th February 2021. The energy range chosen is $100 \mathrm{MeV}-500 \mathrm{GeV}$, divided into 8 logarithmically spaced bins per energy decade. The $\gamma$-ray data are filtered for good time intervals with DATA_QUAL $>0$ and the recommended instrument configuration for science LAT_CONFIG $==1$. We use photons belonging to the SOURCE class (evclass $=128)$ and split the data set into three energy components: i) $100-300 \mathrm{MeV}$, ii) $300-1000 \mathrm{MeV}$, and iii) $1-500 \mathrm{GeV}$, for which we consider events belonging to different types of point spread function, given by evtype $=48,56$, and 3, respectively. To reduce contamination from the Earth limb, we adopt specific maximum zenith angle cuts of $85^{\circ}, 90^{\circ}$, and $105^{\circ}$ for components i), ii) and iii), respectively.

The Galactic and extragalactic background emissions are modeled with the latest interstellar emission model ${ }^{4}$ gll_iem_v07 and with the isotropic spectral template $i s o \_P 8 R 3 \_S O U R C E \_V 2 \_v 1$. We perform the data analysis with Fermitools ${ }^{5}$ v1.2.23 and fermipy ${ }^{6}$ v0.17.4, by means of a binned likelihood analysis and using MINUIT as the minimizer. In the model of the ROI, we include $\gamma$-ray sources listed in 4FGL as well as all sources found with the fermipy function find_sources () and located up to $12^{\circ}$ from the target. Our target is modeled with a single power law.

\section{Discussion and conclusions}

The blazar NVSS J065844+063711 is the only $\gamma$-ray source found within the IceCube neutrino error region. We cross-checked the WISE Blazar-Like Radio-Loud Sources catalog [15] and RomaBZCat [16] for the presence of blazar-like sources within the neutrino $90 \%$ localization region and

4https://fermi.gsfc.nasa.gov/ssc/data/access/lat/BackgroundModels.html

5https://fermi.gsfc.nasa.gov/ssc/data/analysis/software/

6https://fermipy . readthedocs . io/en/latest/index.html 


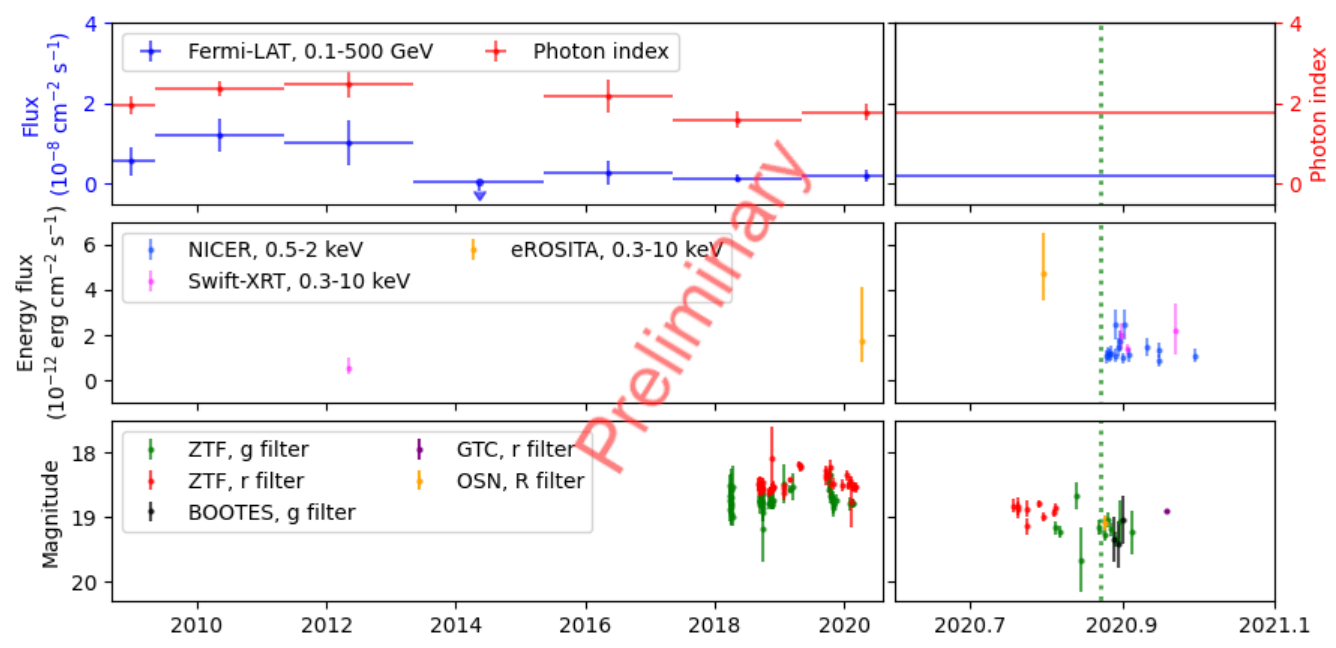

Figure 2: Multiwavelength light curves of NVSS J065844+063711. No flaring activity is observed around the neutrino arrival (green dotted line). The left panels show the observations performed between August 2008 and July 2020, while the right panels show an expanded time scale between July 2020 and February 2021. In the top panels, we show the Fermi-LAT fluxes and photon indexes. For the middle panels, we show the X-ray observations performed with NICER, eROSITA and Swift-XRT. In the bottom panels, we show the optical observations collected with ZTF, BOOTES, GTC and OSN. For more details on the data collection and analysis, we refer the reader to [22].

found none beside NVSS J065844+063711. This is a BL Lac object of unknown redshift ${ }^{7}$ at coordinates R.A. $=104.6876^{\circ}$ and Dec. $=6.6199^{\circ}$ [18]. It lies $0.81^{\circ}$ away from the best-fit localization of IceCube-201114A, i.e., well within the $90 \%$ neutrino positional uncertainty region.

Gamma-ray emission positionally consistent with this object was first reported in the FermiLAT First Source Catalog [1FGL J0658.5+0641; 19] as an unassociated $\gamma$-ray source. The association with NVSS J065844+063711 was established in the Fermi-LAT Third Source Catalog [3FGL; 17]. During the first four years of LAT operations, this source displayed a soft power-law spectrum with a photon index $\Gamma \sim 2.5 \pm 0.1$, according to the 3FGL. In the 4FGL, built integrating the first eight years of LAT data, the source displayed a somewhat harder photon index of $\Gamma \sim 2.2 \pm 0.1$ and had a variability index of 10 , consistent with no significant variability detected in $\gamma$-ray on year timescales [see 4FGL; 14].

The light curves in Figure 2 show overall minor variability. The arrival time of IceCube201114A (highlighted by the green dotted line) coincides with a quiescent state of NVSS J065844 +063711 , where no remarkable enhanced activity was detected at $\gamma$-ray, X-ray or optical wavelengths. The spectral energy distribution (SED) is shown in Figure 3. It is consistent with an HSP blazar with the low-energy bump peaking at $v=3 \times 10^{15} \mathrm{~Hz}(\sim 4 \mathrm{eV})$ [20]. NVSS J065844+063711 is also included in the Fermi-LAT Third Hard Source Catalog, being associated with 3FHL J0658.5+0636 [21]. The highest energy photon reported in the 3FHL consistent with this object is a $\sim 61 \mathrm{GeV}$ event. In January 2018, i.e., nearly two years prior to the arrival of IceCube-201114A, a VHE photon with a $155 \mathrm{GeV}$ energy was detected with a probability of $\sim 95 \%$ of being associated with

\footnotetext{
7https://web.oapd.inaf.it/zbllac/refindex.html
} 


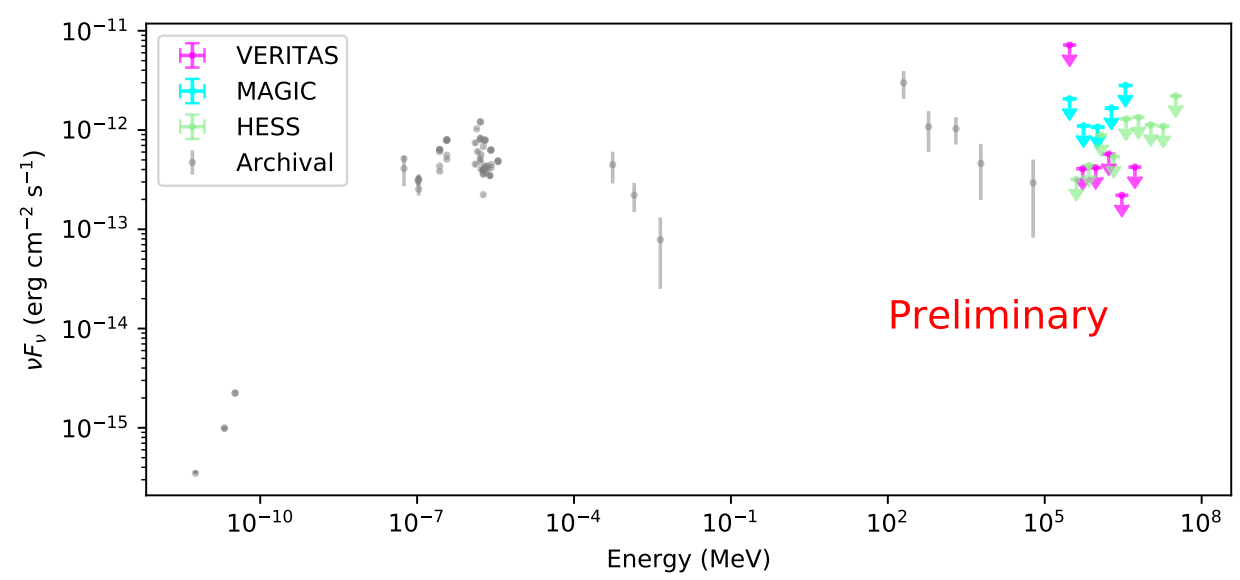

Figure 3: The spectral energy distribution of NVSS J065844+063711. Archival observations collected from the ASDC SED Builder are shown in gray and illustrate the historical flux level of this blazar. The colored data points indicate quasi-simultaneous observations collected with VHE telescopes. For more details on the data collection and analysis, we refer the reader to [22].

this blazar [13]. This VHE photon was accompanied by three other high-energy (i.e., > $10 \mathrm{GeV}$ ) photons within a $\sim 1$-year period. However, observations by the H.E.S.S., MAGIC and VERITAS telescopes did not lead to the detection of significant VHE emission from NVSS J065844+063711.

Previously, the only VHE $\gamma$-ray blazar found within the $90 \%$ confidence region of a wellreconstructed IceCube high-energy neutrino event is TXS 0506+056. NVSS J065844+063711, the second only candidate VHE emitter potentially associated with an astrophysical neutrino, may provide crucial information about the hadronic processes at work in the jets of active galactic nuclei and clues on the origin of cosmic neutrinos. To check if this neutrino-blazar association is by chance, a complete statistical analysis will be undertaken in [22].

\section{Acknowledgements}

SB acknowledges financial support by the European Research Council for the ERC Starting grant MessMapp, under contract no. 949555. The Fermi-LAT Collaboration acknowledges support for LAT development, operation and data analysis from NASA and DOE (United States), CEA/Irfu and IN2P3/CNRS (France), ASI and INFN (Italy), MEXT, KEK, and JAXA (Japan), and the K.A. Wallenberg Foundation, the Swedish Research Council and the National Space Board (Sweden). Science analysis support in the operations phase from INAF (Italy) and CNES (France) is also gratefully acknowledged. This work performed in part under DOE Contract DE-AC02-76SF00515. This research is partly based on observations with the 100-m telescope of the MPIfR (Max-Planck-Institut für Radioastronomie) at Effelsberg.

The following persons contributed to the H.E.S.S. analyses: H. Ashkar (IRFU), Y. Becherini (LNU), M. Cerruti (APC), C. Hoischen (Uni. Potsdam), R. Konno (Desy), G. Kukec Mezek (LNU), F. Schüssler (IRFU), M. Senniappan (LNU). The members of the H.E.S.S. Collaboration gratefully acknowledge financial support from the agencies and organizations listed here. FS acknowledges support for this work by the Programme National des Hautes Energies of CNRS/INSU with INP and IN2P3, co-funded by CEA and CNES. The following persons contributed to the MAGIC analyses and campaign organization: Alessio 
Berti (MPP), Jorge Otero Santos (IAC), Konstancja Satalecka (DESY) and Elisa Bernardini (Uni. Padua). The members of the MAGIC Collaboration gratefully acknowledge financial support from the agencies and organizations listed here. The members of the VERITAS Collaboration gratefully acknowledge financial support from the agencies and organizations listed here. The ZTF collaboration acknowledges support from institutes and agencies listed here.

\section{References}

[1] Aartsen, M. G. et al. First observation of PeV-energy neutrinos with IceCube. PRL, 111, 2 , 2013.

[2] Aartsen, M. G. et al. Evidence for high-energy extraterrestrial neutrinos at the IceCube detector. Science, 342, 6161, 2013.

[3] Mészáros, P. Astrophysical sources of high-energy neutrinos in the IceCube era. Annual Review of Nuclear and Particle Science, 67, 45, 2017.

[4] IceCube Collaboration. Multimessenger observations of a flaring blazar coincident with highenergy neutrino IceCube-170922A. Science, 361, 6398, 2018.

[5] Aartsen, M. at al. Neutrino emission from the direction of the blazar TXS 0506+056 prior to the IceCube-170922A alert. Science, 361, 6398, 2018.

[6] Padovani, P. et al. Dissecting the region around IceCube-170922A: the blazar TXS 0506+056 as the first cosmic neutrino source. MNRAS, 480, 1, 2018.

[7] Garrappa, S. et al. Investigation of two Fermi-LAT gamma-ray blazars coincident with highenergy neutrinos detected by IceCube. ApJ, 880, 2, 2019.

[8] Mücke, A. and Protheroe, R.J. A proton synchrotron blazar model for flaring in Markarian 501. Astroparticle Physics, 15, 1, 2001.

[9] Mücke, A. et al. BL Lac objects in the synchrotron proton blazar mode. Astroparticle Physics, $18,6,2003$.

[10] Keivani, A. et al. A multimessenger picture of the flaring blazar TXS 0506+ 056: Implications for high-energy neutrino emission and cosmic-ray acceleration. ApJ, 864, 1, 2018.

[11] Cerruti, M. et al. Leptohadronic single-zone models for the electromagnetic and neutrino emission of TXS 0506+ 056. MNRAS Letters, 483, 1, 2019.

[12] Garrappa, S. \& Buson, S. Fermi-LAT Gamma-ray Observations of IceCube-201114A. The Astronomer's Telegram, 14188, 1, 2020.

[13] Buson, S., Garrappa, S. and Cheung, C.C. Fermi-LAT evidence for VHE emission from NVSS J065844+063711. The Astronomer's Telegram, 14200, 1, 2020.

[14] Abdollahi, S. et al. Fermi large area telescope fourth source catalog. ApJS, 247, 1, 2020. 
[15] D'Abrusco, R. et al. Two new catalogs of blazar candidates in the WISE infrared sky. ApJS, 242, 1, 2019.

[16] Massaro, E. et al. The 5th edition of the Roma-BZCAT. A short presentation. Ap\&SS, 357, 1, 2015.

[17] Acero, F. et al. Fermi large area telescope third source catalog. ApJS, 218, 2, 2015.

[18] Landoni, M. et al. ZBLLAC: A Spectroscopic Database of BL Lacertae Objects. ApJS, 250, 2,2020 .

[19] Abdo, A. et al. Fermi Large Area Telescope First Source Catalog. ApJS, 188, 2, 2010.

[20] Chang, Y.-L., et al. The 3HSP catalogue of extreme and high-synchrotron-peaked blazars. A\& A, 632, A77, 2019.

[21] Ajello, M., et al. 3FHL: The Third Catalog of Hard Fermi-LAT Sources. ApJS, 232, 2, 2017.

[22] de Menezes, R., et al. in prep.

[23] De Naurois, M. and Rolland, L., A high performance likelihood reconstruction of $\gamma$-rays for imaging atmospheric Cherenkov telescopes, APP, 5, 32, 2009.

[24] Parsons, RD and Hinton, J., A Monte Carlo template based analysis for air-Cherenkov arrays, APP, 56, 2014.

[25] Berge, D. and Funk, S. and Hinton, J., Background modelling in very-high-energy gamma-ray astronomy, AAP, 466, 2007.

[26] R. Zanin et al. Proceedings, 33rd International Cosmic Ray Conference (ICRC2013), p2937 July 2013.

[27] Maier, G. and Holder, J., Eventdisplay: An Analysis and Reconstruction Package for Groundbased Gamma-ray Astronomy, 35th International Cosmic Ray Conference (ICRC2017), p. 747. Jan., 2017.

[28] Cogan., P. VEGAS, the VERITAS Gamma-ray Analysis Suite, 30th International Cosmic Ray Conference (ICRC2007), pp. 1385-1388. Jan., 2008.

[29] Satalecka, K. Searching for VHE gamma-ray emission associated with IceCube neutrino alerts using FACT, H.E.S.S., MAGIC, and VERITAS, 37th International Cosmic Ray Conference (ICRC2021)

[30] Coleiro, A. and Dornic, D. IceCube-201114A: No neutrino counterpart candidates in ANTARES search, GCN 28890, 2020.

[31] Rolke, Wolfgang A., Angel M. Lopez, and Jan Conrad. Limits and confidence intervals in the presence of nuisance parameters., Nuclear Instruments and Methods in Physics Research Section A: Accelerators, Spectrometers, Detectors and Associated Equipment 551.2-3 (2005): 493-503. 


\section{Full Authors List: Fermi-LAT, H.E.S.S., MAGIC, VERITAS, ZTF, and TELAMON Collaborations}

Raniere de Menezes ${ }^{a}$, Sara Buson ${ }^{b}$, Simone Garrappa ${ }^{e}$, Andrea Gokus ${ }^{b, n}$, Matthias Kadler ${ }^{b}$, Teddy Cheung ${ }^{t}$, Marcello Giroletti $^{u}$, Marco Ajello $^{x}$, Francesco Massaro ${ }^{i}$, Harold Peña-Herazo ${ }^{y}$, Fabian Schüssler ${ }^{c}$, Elisa Bernardini ${ }^{d}$, Konstancja Satalecka $^{e}$, Alessandro Paggi ${ }^{i, j, k}$, Andrea Tramacere ${ }^{l}$, Charlotte Ward ${ }^{m}$, Suvi Gezari ${ }^{z}$, Frank J. Masci ${ }^{f}$, Richard Walters ${ }^{g}$, Steven Hämmerich ${ }^{n}$, Joern Wilms ${ }^{n}$, Werner Colmar ${ }^{p}$, Uwe Bach $^{o}$, Florian Eppel ${ }^{b}$, Jonas Heßdörfer ${ }^{b}$, Alex Kraus ${ }^{o}$, Georgios Filippos Paraschos ${ }^{o}$, Javier Moldón $^{q}$, Miguel Pérez-Torres ${ }^{q}$, Iván $\operatorname{Agudo}^{q}$, Giacomo Bonnoliq ${ }^{q}$, Alberto J. Castro-Tirado $q$, Youdong $\mathrm{Hu}^{q}$, Maria D. CaballeroGarcia $^{q}$, Emilio Fernandez-Garcia $^{q}$, Ruben Sanchez-Ramirez $^{r}$, Alexis Coleiro ${ }^{s}$, Antoine Kouchner ${ }^{s}$, Cristina Nanci ${ }^{u, v}$, Yong Sheng ${ }^{x}$, and Meenakshi Rajagopal ${ }^{x}$

${ }^{a}$ Universidade de São Paulo, Departamento de Astronomia, Rua do Matão, 1226, São Paulo, SP 05508-090, Brazil.

${ }^{b}$ Institut für Theoretische Physik and Astrophysik, Universität Würzburg, D-97074 Würzburg, Germany.

${ }^{c}$ IRFU, CEA, Université Paris-Saclay, F-91191 Gif-sur-Yvette, France.

${ }^{d}$ University of Padova, Physics \& Astronomy Dep. "G. Galilei", F. Marzolo, 8, 35121, Padova, Italy.

${ }^{e}$ DESY, Platannenallee 6, 15387 Zeuthen, Germany.

$f$ IPAC, California Institute of Technology, 1200 E. California Blvd, Pasadena, CA 91125, USA

${ }^{g}$ Division of Physics, Mathematics, and Astronomy, California Institute of Technology, Pasadena, CA 91125, USA

${ }^{i}$ Dipartimento di Fisica, Università degli Studi di Torino, via Pietro Giuria 1, I-10125 Torino, Italy.

${ }^{j}$ INAF-Osservatorio Astrofisico di Torino, via Osservatorio 20, I-10025 Pino Torinese, Italy.

${ }^{k}$ Istituto Nazionale di Fisica Nucleare, Sezione di Torino, via Pietro Giuria 1, I-10125 Torino, Italy.

${ }^{l}$ Department of Astronomy, University of Geneva, Ch. d'Ècogia 16, 1290, Versoix, Switzerland.

${ }^{m}$ Department of Astronomy, University of Maryland, College Park, MD 20742, USA.

${ }^{n}$ Dr. Karl Remeis-Observatory and Erlangen Centre for Astroparticle Physics, Universität Erlangen-Nürnberg, Sternwartstr. 7, 96049 Bamberg, Germany.

${ }^{\circ}$ Max-Planck-Institut für Radioastronomie, Auf dem Hügel 69, 53121, Bonn, Germany.

${ }^{p}$ Max-Planck-Institut für extraterrestische Physik, Garching, Germany.

${ }^{q}$ Instituto de Astrofísica de Andalucía (CSIC), Glorieta de la Astronomía, s/n, E-18008 Granada, Spain.

${ }^{r}$ INAF, Istituto di Astrofisica e Planetologia Spaziali, via Fosso del Cavaliere 100, I-00133 Rome, Italy.

${ }^{s}$ Université de Paris, CNRS, Astroparticule et Cosmologie, F-75013 Paris, France.

${ }^{t}$ Naval Research Laboratory, Space Science Division, Washington, DC 20375, USA.

${ }^{u}$ INAF Istituto di Radioastronomia, via Gobetti 101, I-40129 Bologna, Italy.

${ }^{v}$ Department of Physics and Astronomy, University of Bologna, Via Gobetti 93/2 - 40129 Bologna, Italy.

${ }^{x}$ Department of Physics and Astronomy, Clemson University, Kinard Lab of Physics, Clemson, SC 29634-0978, USA.

${ }^{y}$ Instituto Nacional de Astrofísica, Óptica y Electrónica, Apartado Postal 51-216, 72000 Puebla, México.

${ }^{z}$ Space Telescope Science Institute, 3700 San Martin Dr., Baltimore, MD 21218, USA. 


\section{Full Authors List: VERITAS Collaboration}

C. B. Adams ${ }^{1}$, A. Archer ${ }^{2}$, W. Benbow ${ }^{3}$, A. Brill ${ }^{1}$, J. H. Buckley ${ }^{4}$, M. Capasso ${ }^{5}$, J. L. Christiansen ${ }^{6}$, A. J. Chromey ${ }^{7}$, M. Errando ${ }^{4}$, A. Falcone ${ }^{8}$, K. A. Farrel1 $1^{9}$, Q. Feng ${ }^{5}$, G. M. Foote ${ }^{10}$, L. Fortson ${ }^{11}$, A. Furniss ${ }^{12}$, A. Gent ${ }^{13}$, G. H. Gillanders ${ }^{14}$, C. Giuri ${ }^{15}$, O. Gueta ${ }^{15}$, D. Hanna ${ }^{16}$, O. Hervet ${ }^{17}$, J. Holder ${ }^{10}$, B. Hona ${ }^{18}$, T. B. Humensky ${ }^{1}$, W. Jin ${ }^{19}$, P. Kaaret ${ }^{20}$, M. Kertzman ${ }^{2}$, T. K. Kleiner ${ }^{15}$, S. Kumar ${ }^{16}$, M. J. Lang ${ }^{14}$, M. Lundy ${ }^{16}$, G. Maier ${ }^{15}$, C. E McGrath ${ }^{9}$, P. Moriarty ${ }^{14}$, R. Mukherjee ${ }^{5}$, D. Nieto ${ }^{21}$, M. Nievas-Rosillo ${ }^{15}$, S. O'Brien ${ }^{16}$, R. A. Ong ${ }^{22}$, A. N. Otte ${ }^{13}$, S. R. Patel ${ }^{15}$, S. Patel ${ }^{20}$, K. Pfrang ${ }^{15}$, M. Pohl ${ }^{23,15}$, R. R. Prado ${ }^{15}$, E. Pueschel ${ }^{15}$, J. Quinn ${ }^{9}$, K. Ragan ${ }^{16}$, P. T. Reynolds ${ }^{24}$, D. Ribeiro ${ }^{1}$, E. Roache ${ }^{3}$, J. L. Ryan ${ }^{22}$, I. Sadeh ${ }^{15}$, M. Santander ${ }^{19}$, G. H. Sembroski ${ }^{25}$, R. Shang ${ }^{22}$, D. Tak ${ }^{15}$, V. V. Vassiliev ${ }^{22}$, A. Weinstein ${ }^{7}$, D. A. Williams ${ }^{17}$, and T. J. Williamson ${ }^{10}$

${ }^{1}$ Physics Department, Columbia University, New York, NY 10027, USA ${ }^{2}$ Department of Physics and Astronomy, DePauw University, Greencastle, IN 46135-0037, USA ${ }^{3}$ Center for Astrophysics | Harvard \& Smithsonian, Cambridge, MA 02138, USA ${ }^{4}$ Department of Physics, Washington University, St. Louis, MO 63130, USA ${ }^{5}$ Department of Physics and Astronomy, Barnard College, Columbia University, NY 10027, USA ${ }^{6}$ Physics Department, California Polytechnic State University, San Luis Obispo, CA 94307, USA ${ }^{7}$ Department of Physics and Astronomy, Iowa State University, Ames, IA 50011, USA ${ }^{8}$ Department of Astronomy and Astrophysics, 525 Davey Lab, Pennsylvania State University, University Park, PA 16802, USA ${ }^{9}$ School of Physics, University College Dublin, Belfield, Dublin 4, Ireland ${ }^{10}$ Department of Physics and Astronomy and the Bartol Research Institute, University of Delaware, Newark, DE 19716, USA ${ }^{11}$ School of Physics and Astronomy, University of Minnesota, Minneapolis, MN 55455, USA ${ }^{12}$ Department of Physics, California State University - East Bay, Hayward, CA 94542, USA ${ }^{13}$ School of Physics and Center for Relativistic Astrophysics, Georgia Institute of Technology, 837 State Street NW, Atlanta, GA 30332-0430 ${ }^{14}$ School of Physics, National University of Ireland Galway, University Road, Galway, Ireland ${ }^{15}$ DESY, Platanenallee 6, 15738 Zeuthen, Germany ${ }^{16}$ Physics Department, McGill University, Montreal, QC H3A 2T8, Canada ${ }^{17}$ Santa Cruz Institute for Particle Physics and Department of Physics, University of California, Santa Cruz, CA 95064, USA ${ }^{18}$ Department of Physics and Astronomy, University of Utah, Salt Lake City, UT 84112, USA ${ }^{19}$ Department of Physics and Astronomy, University of Alabama, Tuscaloosa, AL 35487, USA ${ }^{20}$ Department of Physics and Astronomy, University of Iowa, Van Allen Hall, Iowa City, IA 52242, USA ${ }^{21}$ Institute of Particle and Cosmos Physics, Universidad Complutense de Madrid, 28040 Madrid, Spain 22 Department of Physics and Astronomy, University of California, Los Angeles, CA 90095, USA ${ }^{23}$ Institute of Physics and Astronomy, University of Potsdam, 14476 Potsdam-Golm, Germany ${ }^{24}$ Department of Physical Sciences, Munster Technological University, Bishopstown, Cork, T12 P928, Ireland ${ }^{25}$ Department of Physics and Astronomy, Purdue University, West Lafayette, IN 47907, USA

\section{Full Authors List: MAGIC Collaboration}

V. A. Acciari ${ }^{1}$, S. Ansoldi ${ }^{2,41}$, L. A. Antonelli ${ }^{3}$, A. Arbet Engels ${ }^{4}$, M. Artero ${ }^{5}$, K. Asano ${ }^{6}$, D. Baack ${ }^{7}$, A. Babié ${ }^{8}$, A. Baquero ${ }^{9}$, U. Barres de Almeida ${ }^{10}$, J. A. Barrio ${ }^{9}$, I. Batković ${ }^{11}$, J. Becerra González ${ }^{1}$, W. Bednarek ${ }^{12}$, L. Bellizzi ${ }^{13}$, E. Bernardini ${ }^{14}$, M. Bernardos ${ }^{11}$, A. Berti ${ }^{15}$, J. Besenrieder ${ }^{15}$, W. Bhattacharyya ${ }^{14}$, C. Bigongiari ${ }^{3}$, A. Biland ${ }^{4}$, O. Blanch ${ }^{5}$, H. Bökenkamp ${ }^{7}$, G. Bonnoli ${ }^{16}$, Ž Bošnjak $^{8}$, G. Busetto ${ }^{11}$, R. Carosi ${ }^{17}$, G. Ceribella ${ }^{15}$, M. Cerruti ${ }^{18}$, Y. Chai ${ }^{15}$, A. Chilingarian ${ }^{19}$, S. Cikota ${ }^{8}$, S. M. Colak ${ }^{5}$, E. Colombo ${ }^{1}$, J. L. Contreras ${ }^{9}$, J. Cortina ${ }^{20}$, S. Covino ${ }^{3}$, G. D’Amico ${ }^{15,42}$, V. D’Elia ${ }^{3}$, P. Da Vela ${ }^{17,43}$, F. Dazzi ${ }^{3}$, A. De Angelis ${ }^{11}$, B. De Lotto ${ }^{2}$, M. Delfino ${ }^{5,44}$, J. Delgado ${ }^{5,44}$, C. Delgado $M_{e n d e z}{ }^{20}$, D. Depaoli ${ }^{21}$, F. Di Pierro ${ }^{21}$, L. Di Venere ${ }^{22}$, E. Do Souto Espiñeira ${ }^{5}$, D. Dominis Prester $^{23}$, A. Donini ${ }^{2}$, D. Dorner ${ }^{24}$, M. Doro ${ }^{11}$, D. Elsaesser ${ }^{7}$, V. Fallah Ramazani ${ }^{25,45}$, A. Fattorini ${ }^{7}$, M. V. Fonseca ${ }^{9}$, L. Font ${ }^{26}$, C. Fruck ${ }^{15}$, S. Fukami ${ }^{6}$, Y. Fukazawa ${ }^{27}$, R. J. García López ${ }^{1}$, M. Garczarczyk ${ }^{14}$, S. Gasparyan ${ }^{28}$, M. Gaug ${ }^{26}$, N. Giglietto ${ }^{22}$, F. Giordano ${ }^{22}$, P. Gliwny ${ }^{12}$, N. Godinovic ${ }^{29}$, J. G. Green ${ }^{3}$, D. Green ${ }^{15}$, D. Hadasch ${ }^{6}$, A. Hahn ${ }^{15}$, L. Heckmann ${ }^{15}$, J. Herrera ${ }^{1}$,

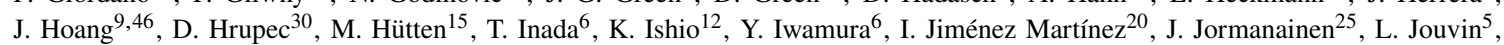
M. Karjalainen ${ }^{1}$, D. Kerszberg ${ }^{5}$, Y. Kobayashi' ${ }^{6}$, H. Kubo ${ }^{31}$, J. Kushida ${ }^{32}$, A. Lamastra ${ }^{3}$, D. Lelas ${ }^{29}$, F. Leone ${ }^{3}$, E. Lindfors ${ }^{25}$, L. Linhoff ${ }^{7}$, S. Lombardi ${ }^{3}$, F. Longo ${ }^{2,47}$, R. López-Coto ${ }^{11}$, M. López-Moya ${ }^{9}$, A. López-Oramas ${ }^{1}$, S. Loporchio ${ }^{22}$, B. Machado de Oliveira Fraga $^{10}$, C. Maggio ${ }^{26}$, P. Majumdar ${ }^{33}$, M. Makariev ${ }^{34}$, M. Mallamaci ${ }^{11}$, G. Maneva ${ }^{34}$, M. Manganaro ${ }^{23}$, K. Mannheim ${ }^{24}$, L. Maraschi ${ }^{3}$, M. Mariotti ${ }^{11}$, M. Martínez ${ }^{5}$, D. Mazin ${ }^{6,48}$, S. Menchiari ${ }^{13}$, S. Mender ${ }^{7}$, S. Mićanović23 ${ }^{23}$ D. Miceli ${ }^{2,49}$, T. Miener $^{9}$, J. M. Miranda ${ }^{13}$, R. Mirzoyan ${ }^{15}$, E. Molina ${ }^{18}$, A. Moralejo ${ }^{5}$, D. Morcuende ${ }^{9}$, V. Moreno ${ }^{26}$, E. Moretti ${ }^{5}$, T. Nakamori ${ }^{35}$, L. Nava ${ }^{3}$, V. Neustroev ${ }^{36}$, C. Nigro ${ }^{5}$, K. Nilsson ${ }^{25}$, K. Nishijima ${ }^{32}$, K. Noda $^{6}$, S. Nozaki ${ }^{31}$, Y. Ohtani ${ }^{6}$, T. Oka ${ }^{31}$, J. Otero-Santos ${ }^{1}$, S. Paiano ${ }^{3}$, M. Palatiello ${ }^{2}$, D. Paneque ${ }^{15}$, R. Paoletti ${ }^{13}$, J. M. Paredes ${ }^{18}$, L. Pavletić ${ }^{23}$, P. Peñil ${ }^{9}$, M. Persic ${ }^{2,50}$, M. Pihet ${ }^{15}$, P. G. Prada Moroni ${ }^{17}$, E. Prandini ${ }^{11}$, C. Priyadarshi ${ }^{5}$, I. Puljak ${ }^{29}$, W. Rhode ${ }^{7}$, M. Ribó ${ }^{18}$, J. Rico ${ }^{5}$, C. Righi ${ }^{3}$, A. Rugliancich ${ }^{17}$, N. Sahakyan ${ }^{28}$, T. Saito ${ }^{6}$, S. Sakurai ${ }^{6}$, K. Satalecka ${ }^{14}$, F. G. Saturni ${ }^{3}$, B. Schleicher ${ }^{24}$, K. Schmidt ${ }^{7}$, T. Schweizer ${ }^{15}$, J. Sitarek ${ }^{12}$, I. Šnidaric ${ }^{37}$, D. Sobczynska ${ }^{12}$, A. Spolon ${ }^{11}$, A. Stamerra ${ }^{3}$, J. Strišković ${ }^{30}$, D. Strom ${ }^{15}$, M. Strzys ${ }^{6}$, Y. Suda ${ }^{27}$, T. Surić ${ }^{37}$, M. Takahashi ${ }^{6}$, R. Takeishi ${ }^{6}$, F. Tavecchio $^{3}$, P. Temnikov ${ }^{34}$, T. Terzic ${ }^{23}$, M. Teshima ${ }^{15,51}$, L. Tosti ${ }^{38}$, S. Truzzi ${ }^{13}$, A. Tutone ${ }^{3}$, S. Ubach ${ }^{26}$, J. van Scherpenberg ${ }^{15}$, G. Vanzo $^{1}$, M. Vazquez Acosta ${ }^{1}$, S. Ventura ${ }^{13}$, V. Verguilov ${ }^{34}$, C. F. Vigorito ${ }^{21}$, V. Vitale ${ }^{39}$, I. Vovk $^{6}$, M. Will ${ }^{15}$, C. Wunderlich ${ }^{13}$, T. Yamamoto $^{40}$, and D. Zaric ${ }^{29}$

\footnotetext{
${ }^{1}$ Instituto de Astrofísica de Canarias and Dpto. de Astrofísica, Universidad de La Laguna, E-38200, La Laguna, Tenerife, Spain ${ }^{2}$ Università di Udine and INFN Trieste, I-33100 Udine, Italy ${ }^{3}$ National Institute for Astrophysics (INAF), I-00136 Rome, Italy ${ }^{4}$ ETH Zürich, CH-8093 Zürich, Switzerland ${ }^{5}$ Institut de Física d'Altes Energies (IFAE), The Barcelona Institute of Science and Technology (BIST), E-08193 Bellaterra (Barcelona), Spain ${ }^{6}$ Japanese MAGIC Group: Institute for Cosmic Ray Research (ICRR), The University of Tokyo, Kashiwa, 277-8582 Chiba, Japan ${ }^{7}$ Technische Universität Dortmund, D-44221 Dortmund, Germany ${ }^{8}$ Croatian MAGIC
} 
Group: University of Zagreb, Faculty of Electrical Engineering and Computing (FER), 10000 Zagreb, Croatia ${ }^{9}$ IPARCOS Institute and EMFTEL Department, Universidad Complutense de Madrid, E-28040 Madrid, Spain ${ }^{10}$ Centro Brasileiro de Pesquisas Físicas (CBPF), 22290-180 URCA, Rio de Janeiro (RJ), Brazil ${ }^{11}$ Università di Padova and INFN, I-35131 Padova, Italy ${ }^{12}$ University of Lodz, Faculty of Physics and Applied Informatics, Department of Astrophysics, 90-236 Lodz, Poland ${ }^{13}$ Università di Siena and INFN Pisa, I-53100 Siena, Italy ${ }^{14}$ Deutsches Elektronen-Synchrotron (DESY), D-15738 Zeuthen, Germany ${ }^{15}$ Max-Planck-Institut für Physik, D-80805 München, Germany ${ }^{16}$ Instituto de Astrofísica de Andalucía-CSIC, Glorieta de la Astronomía s/n, 18008, Granada, Spain ${ }^{17}$ Università di Pisa and INFN Pisa, I-56126 Pisa, Italy ${ }^{18}$ Universitat de Barcelona, ICCUB, IEEC-UB, E-08028 Barcelona, Spain ${ }^{19}$ Armenian MAGIC Group: A. Alikhanyan National Science Laboratory, 0036 Yerevan, Armenia ${ }^{20}$ Centro de Investigaciones Energéticas, Medioambientales y Tecnológicas, E-28040 Madrid, Spain ${ }^{21}$ INFN MAGIC Group: INFN Sezione di Torino and Università degli Studi di Torino, I-10125 Torino, Italy ${ }^{22}$ INFN MAGIC Group: INFN Sezione di Bari and Dipartimento Interateneo di Fisica dell'Università e del Politecnico di Bari, I-70125 Bari, Italy ${ }^{23}$ Croatian MAGIC Group: University of Rijeka, Department of Physics, 51000 Rijeka, Croatia ${ }^{24}$ Universität Würzburg, D-97074 Würzburg, Germany ${ }^{25}$ Finnish MAGIC Group: Finnish Centre for Astronomy with ESO, University of Turku, FI-20014 Turku, Finland ${ }^{26}$ Departament de Física, and CERES-IEEC, Universitat Autònoma de Barcelona, E-08193 Bellaterra, Spain 27 Japanese MAGIC Group: Physics Program, Graduate School of Advanced Science and Engineering, Hiroshima University, 7398526 Hiroshima, Japan ${ }^{28}$ Armenian MAGIC Group: ICRANet-Armenia at NAS RA, 0019 Yerevan, Armenia ${ }^{29}$ Croatian MAGIC Group: University of Split, Faculty of Electrical Engineering, Mechanical Engineering and Naval Architecture (FESB), 21000 Split, Croatia ${ }^{30}$ Croatian MAGIC Group: Josip Juraj Strossmayer University of Osijek, Department of Physics, 31000 Osijek, Croatia ${ }^{31}$ Japanese MAGIC Group: Department of Physics, Kyoto University, 606-8502 Kyoto, Japan ${ }^{32}$ Japanese MAGIC Group: Department of Physics, Tokai University, Hiratsuka, 259-1292 Kanagawa, Japan ${ }^{33}$ Saha Institute of Nuclear Physics, HBNI, 1/AF Bidhannagar, Salt Lake, Sector-1, Kolkata 700064, India ${ }^{34}$ Inst. for Nucl. Research and Nucl. Energy, Bulgarian Academy of Sciences, BG-1784 Sofia, Bulgaria ${ }^{35}$ Japanese MAGIC Group: Department of Physics, Yamagata University, Yamagata 990-8560, Japan ${ }^{36}$ Finnish MAGIC Group: Astronomy Research Unit, University of Oulu, FI-90014 Oulu, Finland ${ }^{37}$ Croatian MAGIC Group: Ruđer Bošković Institute, 10000 Zagreb, Croatia ${ }^{38}$ INFN MAGIC Group: INFN Sezione di Perugia, I-06123 Perugia, Italy ${ }^{39}$ INFN MAGIC Group: INFN Roma Tor Vergata, I-00133 Roma, Italy ${ }^{40}$ Japanese MAGIC Group: Department of Physics, Konan University, Kobe, Hyogo 658-8501, Japan ${ }^{41}$ also at International Center for Relativistic Astrophysics (ICRA), Rome, Italy ${ }^{42}$ now at Department for Physics and Technology, University of Bergen, NO-5020, Norway ${ }^{43}$ now at University of Innsbruck ${ }^{44}$ also at Port d'Informació Científica (PIC), E-08193 Bellaterra (Barcelona), Spain ${ }^{45}$ now at Ruhr-Universität Bochum, Fakultät für Physik und Astronomie, Astronomisches Institut (AIRUB), 44801 Bochum, Germany ${ }^{46}$ now at Department of Astronomy, University of California Berkeley, Berkeley CA 94720 47 also at Dipartimento di Fisica, Università di Trieste, I-34127 Trieste, Italy ${ }^{48}$ Max-Planck-Institut für Physik, D-80805 München, Germany 49 now at Laboratoire d'Annecy de Physique des Particules (LAPP), CNRS-IN2P3, 74941 Annecy Cedex, France ${ }^{50}$ also at INAF Trieste and Dept. of Physics and Astronomy, University of Bologna, Bologna, Italy ${ }^{51}$ Japanese MAGIC Group: Institute for Cosmic Ray Research (ICRR), The University of Tokyo, Kashiwa, 277-8582 Chiba, Japan 